\title{
The cost-effectiveness of digital breast tomosynthesis in a population breast cancer screening program
}

\author{
Jing Wang ${ }^{1} \cdot$ Xuan-Anh Phi ${ }^{1}$ Marcel J. W. Greuter ${ }^{2}$ - Alicja M. Daszczuk ${ }^{1,3} \cdot$ Talitha L. Feenstra $^{4} \cdot$ Ruud M. Pijnappel $^{4}$. \\ Karin M. Vermeulen ${ }^{1}$. Nico Buls ${ }^{3}$ • Nehmat Houssami ${ }^{5}$. Wenli Lu ${ }^{6}$. Geertruida H. de Bock ${ }^{1}$
}

Received: 18 September 2019 / Revised: 17 February 2020 / Accepted: 13 March 2020 / Published online: 7 May 2020

(C) The Author(s) 2020

\begin{abstract}
Objectives To evaluate at which sensitivity digital breast tomosynthesis (DBT) would become cost-effective compared to digital mammography (DM) in a population breast cancer screening program, given a constant estimate of specificity.

Methods In a microsimulation model, the cost-effectiveness of biennial screening for women aged 50-75 was simulated for three scenarios: DBT for women with dense breasts and DM for women with fatty breasts (scenario 1), DBT for the whole population (scenario 2) or maintaining DM screening (reference). For DM, sensitivity was varied depending on breast density from 65 to $87 \%$, and for DBT from 65 to $100 \%$. The specificity was set at $96.5 \%$ for both DM and DBT. Direct medical costs were considered, including screening, biopsy and treatment costs. Scenarios were considered to be cost-effective if the incremental cost-effectiveness ratio (ICER) was below €20,000 per life year gain (LYG).

Results For both scenarios, the ICER was more favourable at increasing DBT sensitivity. Compared with DM screening, 0.8$10.2 \%$ more LYGs were found when DBT sensitivity was at least $75 \%$ for scenario 1 , and $4.7-18.7 \%$ when DBT sensitivity was at least $80 \%$ for scenario 2 . At $€ 96$ per DBT, scenario 1 was cost-effective at a DBT sensitivity of at least $90 \%$, and at least $95 \%$ for scenario 2. At $€ 80$ per DBT, these values decreased to $80 \%$ and $90 \%$, respectively.

Conclusion DBT is more likely to be a cost-effective alternative to mammography in women with dense breasts. Whether DBT could be cost-effective in a general population highly depends on DBT costs.

\section{Key Points}

- DBT could be a cost-effective screening modality for women with dense breasts when its sensitivity is at least $90 \%$ at a maximum cost per screen of $€ 96$.

- DBT has the potential to be cost-effective for screening all women when sensitivity is at least $90 \%$ at a maximum cost per screen of $€ 80$.

- Whether DBT could be used as an alternative to mammography for screening all women is highly dependent on the cost of DBT per screen.
\end{abstract}

Keywords Breast neoplasms $\cdot$ Mammography $\cdot$ Tomosynthesis $\cdot$ Mass screening $\cdot$ Cost-benefit analysis

Electronic supplementary material The online version of this article (https://doi.org/10.1007/s00330-020-06812-x) contains supplementary material, which is available to authorized users.

Jing Wang

j.wang@umcg.nl

1 Department of Epidemiology, University of Groningen, University Medical Center Groningen, Groningen, The Netherlands

2 Department of Radiology, University of Groningen, University Medical Center Groningen, Groningen, The Netherlands
3 Department of Radiology, Vrije Universiteit Brussel, Universitair Ziekenhuis Brussel, Brussels, Belgium

4 Department of Radiology, University Medical Center Utrecht, Utrecht University, Utrecht, The Netherlands

5 Sydney School of Public Health, Faculty of Medicine and Health, University of Sydney, Sydney, Australia

6 Department of Epidemiology and Health Statistics, Tianjin Medical University, Tianjin, China 


$\begin{array}{ll}\text { Abbreviations } \\ \text { CDR } & \begin{array}{l}\text { Cancer detection rate } \\ \text { CHEERS }\end{array} \\ & \begin{array}{l}\text { Consolidated Health Economic } \\ \text { Evaluation Reporting Standards }\end{array} \\ \text { DBT } & \text { Digital breast tomosynthesis } \\ \text { DM } & \text { Digital mammography } \\ \text { ICER } & \text { Incremental cost-effectiveness ratio } \\ \text { LYG } & \text { Life year gained } \\ \text { SE } & \text { Standard error } \\ \text { SiMRiSc } & \begin{array}{l}\text { The simulation model on radiation } \\ \text { risk and breast cancer screening }\end{array}\end{array}$

\section{Introduction}

Breast cancer is one of the leading causes of death among women, and the most common cancer in womenapproximately 1 in 8 women will develop breast cancer in their lifetime [1]. National mammography screening programs have been introduced in most European and developed countries for several decades. The key benefit of regular mammography screening is that it detects breast cancer at an early stage allowing more effective treatment and improved survival [2]. A recent study showed that the incidence of early-stage breast cancer in patients who attend screening regularly is significantly higher than that in patients who did not, suggesting a stage shift to earlier detection with the implementation of regular screening [3]. However, there are also controversies regarding screening. Over-diagnosis and related over-treatment are considered a main drawback of screening, with estimates of over-diagnosis varying from 15 to $30 \%$ [4]. In addition, false-positive results lead to unnecessary biopsies and negative psychological outcomes which can decrease the efficacy and acceptability of screening programs [4]. A key issue is the imperfect sensitivity of digital mammography (DM) which reduces the effectiveness of screening [5].

It is well-documented that mammographic sensitivity decreases with increasing breast density [6]. Moreover, a higher breast density is an additional risk factor for breast cancer, and both false-positive and false-negative interpretations are more likely with dense breasts [7]. Nowadays, digital breast tomosynthesis (DBT) is gaining widespread attention because it improves the detection of cancer particularly in dense breast tissue. When using adjunct DBT in women with DM-negative dense breasts, the ASTOUND-2 trial showed an incremental cancer detection rate (CDR) of 2.83 per 1000 screens [8]. In population-based screening, a recent meta-analysis showed that DBT combined with DM yields a pooled incremental CDR of 2.4 cancers per 1000 screens in biennial screening practice with only a slight increase in recall rate compared to DM alone [9]. With respect to the cost-effectiveness of DBT, the evidence is limited. However, in a study focusing on US population aged 50-74 years, biennial combined DM and
DBT screening for women with dense breasts was reported to be cost-effective if the price of DBT plus DM was below \$226 [10]. Recently, synthesised 2D mammograms from DBT were introduced showing similar sensitivity and specificity with respect to DM [11]. This has made it feasible to use DBT-only acquisition as a stand-alone screening modality instead of combined DBT and DM [12].

In this evolving landscape for population breast screening, we undertook a study aiming to evaluate at what sensitivity DBT could be a cost-effective alternative to DM in a population breast cancer screening program, while the specificity is kept constant. A validated micro-simulation model (SiMRiSc) was applied to simulate biennial population breast cancer screening in The Netherlands for women 50-75 years of age, whereby DBT was applied to replace DM for all women or, alternatively, for women with high breast density, against DM screening as currently practiced. In doing so, we anticipate that the findings from this study could provide healtheconomic evidence to the Dutch program and many organized population screening programs practicing biennial DM screening. However, we need to address other important issues beyond health economics such as risk stratification and estimates of incremental mortality impact, and recall rate should also be taken into consideration before implementing DBT in a population screening program [13].

\section{Material and methods}

This study was reported according to the Consolidated Health Economic Evaluation Reporting Standards (CHEERS) statement [14]. The simulation model on radiation risk and breast cancer screening (SiMRiSc) was applied in the analysis [15-17].

\section{Description of the model and input variables}

SiMRiSc is a micro-simulation Markov model that was previously published and externally validated in the general population and in women with BRCA gene mutations. In summary, women's lifetimes were simulated considering their life expectancy, chance of developing cancer, tumour growth, probability of tumour self-detection and survival probability from breast cancer. If a tumour was present at the screening moment, the chance of detection was determined by the mammographic sensitivity. The sensitivity is a function of breast density which depends on the age of the woman. After breast cancer diagnosis, either by screening or by self-detection, the woman was removed from simulation and the breast cancer age-specific death of the woman was calculated based on life expectancy after diagnosis which depended on tumour size. Also included were mammographic specificity for the introduction of false positives, and the probability of tumour 
Table 1 Input variables and their estimates for the SiMRiSc model

\begin{tabular}{|c|c|c|c|c|c|c|c|}
\hline & \multicolumn{2}{|l|}{ Variable } & \multicolumn{4}{|c|}{ Mean estimate (SD) } & Reference \\
\hline \multirow[t]{10}{*}{ Population } & \multicolumn{2}{|c|}{$\begin{array}{l}\text { Cumulative lifetime breast cancer risk at the age of } 70 \\
\text { Mean onset age of breast cancer }\end{array}$} & \multicolumn{4}{|c|}{$22.6 \%(0.74)$} & \multirow[t]{2}{*}{ [19] } \\
\hline & \multicolumn{2}{|l|}{ SD in onset age of breast cancer } & \multicolumn{4}{|c|}{$21.1(0.93)$} & \\
\hline & \multirow[t]{7}{*}{ Breast density distribution } & Age group & \multicolumn{4}{|c|}{ BI-RADS density } & {$[20-22]$} \\
\hline & & & 1 & 2 & 3 & 4 & \\
\hline & & $<40$ & $5 \%$ & $30 \%$ & $48 \%$ & $17 \%$ & \\
\hline & & $40-50$ & $6 \%$ & $34 \%$ & $47 \%$ & $13 \%$ & \\
\hline & & $50-60$ & $8 \%$ & $50 \%$ & $37 \%$ & $5 \%$ & \\
\hline & & $60-70$ & $15 \%$ & $53 \%$ & $29 \%$ & $3 \%$ & \\
\hline & & $>70$ & $18 \%$ & $54 \%$ & $26 \%$ & $2 \%$ & \\
\hline & \multicolumn{2}{|l|}{ Participation rate } & \multicolumn{4}{|l|}{$80 \%$} & {$[18]$} \\
\hline Tumour induction model & \multicolumn{2}{|l|}{$\begin{array}{l}\text { Excess relative risk of tumour induction } \\
\text { due to radiation per Gy }\end{array}$} & \multicolumn{4}{|c|}{$0.51(0.16)$} & {$[17]$} \\
\hline \multirow[t]{2}{*}{ Tumour growth model } & \multirow[t]{2}{*}{ Tumour doubling time per age group } & $\begin{array}{l}<50 \\
50-70\end{array}$ & \multicolumn{4}{|c|}{$\begin{array}{l}80(28) \text { days } \\
157 \text { (25) days }\end{array}$} & \multirow[t]{2}{*}[23]{} \\
\hline & & $>70$ & 188( & days & & & \\
\hline \multirow{7}{*}{ Digital mammography } & \multirow[t]{3}{*}{ Sensitivity } & & \multicolumn{4}{|c|}{ BI-RADS density } & \multirow[t]{3}{*}[15]{} \\
\hline & & & 1 & 2 & 3 & 4 & \\
\hline & & & $87 \%$ & $84 \%$ & $73 \%$ & $65 \%$ & \\
\hline & \multicolumn{2}{|l|}{ Specificity } & \multicolumn{4}{|c|}{$96.5 \%$} & {$[24]$} \\
\hline & \multicolumn{2}{|l|}{ Cost/screen } & \multicolumn{4}{|l|}{$€ 64$} & {$[25]$} \\
\hline & \multicolumn{2}{|l|}{ Mean glandular dose } & \multicolumn{4}{|c|}{3.0 (1.0) mGy } & {$[17]$} \\
\hline & \multicolumn{2}{|l|}{ Detection threshold } & \multicolumn{4}{|c|}{$5 \mathrm{~mm}$} & {$[26]$} \\
\hline \multirow[t]{5}{*}{ Digital breast tomosynthesis } & Sensitivity* & & $65-10$ & & & & {$[25]$} \\
\hline & Specificity & & $96.5 \%$ & & & & {$[24]$} \\
\hline & Costs/screen & & $€ 96 / €$ & & & & \\
\hline & Mean glandular dose & & $4.0(1$ & $\mathrm{mGy}$ & & & {$[26]$} \\
\hline & Detection threshold & & $5 \mathrm{~mm}$ & & & & {$[17]$} \\
\hline Costs in case of positive finding & Biopsy & & $€ 176$ & & & & {$[27]$} \\
\hline & Treatment (tumour diameter) & $\begin{array}{l}<2 \mathrm{~cm} \\
2-5 \mathrm{~cm}\end{array}$ & $\begin{array}{l}€ 6438 \\
€ 7128\end{array}$ & & & & {$[16]$} \\
\hline & & $>5 \mathrm{~cm}$ & $€ 7701$ & & & & \\
\hline
\end{tabular}

*For sensitivity of DBT, the lower boundary was set at the minimum estimate for the DM sensitivity

$S D=$ standard deviation

induction due to the ionising radiation from mammography. In the model, we assumed an $80 \%$ participation rate and only invasive cancers were considered [18].

The estimates for the model variables were based on published data for population statistics from The Netherlands and the USA, results of systematic searches and published cost estimates (Table 1) [15-27]. The population statistics used in our study included cumulative lifetime risk of breast cancer, mean onset age of breast cancer, breast density distribution and participation rate, which are summarised in Table 1. In this study, all sensitivities and specificities are given as modality sensitivity and modality specificity respectively [28]. Because DBT is a relatively new technique, there are no published data on either breast density averaged sensitivity or sensitivities based on breast density from a screening setting with long follow-up. Therefore, we used a DBT sensitivity that was constant across breast densities, and varied the sensitivity values from 65 to $100 \%$ in steps of $5 \%$, with a lower boundary set at the minimum estimate for DM sensitivity [25]. The DBT specificity was fixed at $96.5 \%$, which was the same as the specificity of DM [24]. The cost of mammography in the Dutch national screening program was determined as the cost of the whole program divided by the number of participants [18]. The base price for DBT was estimated at 1.5 times the DM costs, i.e. $€ 96$ per screen, allowing for a conventional cost-effectiveness estimate, given the higher equipment costs, added digital storage capacity, more expensive reading stations and the double-reading time for DBT [29]. Additionally, a lower estimated cost for DBT was also simulated, which was 1.25 times the DM cost, i.e. $€ 80$ per screen. 
A detailed description of other input variables can be found in our previous studies [15-17].

\section{Screening scenarios}

Three screening scenarios were evaluated. In the first scenario, DBT was used for biennial breast cancer screening only for women with high-density breasts (BI-RADS 4th edition density scores 3 and 4), whereas DM was used for women with non-dense breasts (scenario 1). The breast density distribution was described as a percentage, according to age group, as defined in Table 1. In the second scenario, DBT was used for biennial breast cancer screening for all women aged 5075 (scenario 2). The current breast cancer screening program in The Netherlands, biennial mammography screening with DM for women aged 50-75 years, was simulated as a reference scenario. To make appropriate comparisons among scenarios, we used the same input variables of population statistics, tumour induction model and tumour growth model; however, the modality-related variables such as cost, dose and sensitivity were varied according to the specific modality used.

\section{Outcome of the simulation model}

One hundred thousand women were simulated for all three scenarios in order to minimise the statistical error and keep the computation time within limits (approximately $0.15 \mathrm{~min}$ for each simulation on a PC workstation). Each simulation was repeated 10 times in order to calculate the error of the point estimates. The sensitivity of DBT was varied between 65 and $100 \%$ in steps of $5 \%$. The results from the simulations were reported in terms of the number of screen-detected tumours, the number of interval cancers and the number of life years gained (LYG) for both DBT screening scenarios as compared to the reference. All results and the standard errors (SEs) were calculated based on the outcomes of the simulation repetitions.

\section{Cost-effectiveness analysis}

Only direct medical costs were considered in this study, including the screening costs for different modalities, the treatment costs based on the tumour size and related biopsy costs due to positive screening results. Incremental costeffectiveness ratios (ICER) for both DBT screening scenarios were estimated as the ratio of the additional costs to the additional LYG compared to the reference, and ICER was also estimated between the two DBT scenarios. The ICER threshold was set to $€ 20,000$ per LYG [30]. Discounting is the process of converting future costs to their present value; to reflect the fact that, in general, society prefers to receive benefits sooner rather than later, and pays costs later rather than sooner
[31]. A discount rate of $3 \%$ for both costs and effects was applied allowing for international comparisons [32]. Additionally, a discount rate of $4 \%$ for costs and $1.5 \%$ for health effects (LYG) was applied according to the Dutch guidelines [33]. In the main text, all ICERs are reported as international discounted ICERs; the undiscounted and Dutch discounted ICERs are provided in the supplementary file.

\section{Results}

The reference scenario of DM screening resulted in a cumulative count of 536 screen-detected cancers, 272 interval cancers and 1353 LYG when 10,000 women were screened biennially from age 50 to 74 years. The results based on modelled sensitivity of DBT showed that the effectiveness of both DBT scenarios increased at increased DBT sensitivity (Table 2) as more screen-detected tumours, fewer interval tumours and more LYG were observed. When the sensitivity of DBT was lower than $72 \%$, both DBT scenarios were less effective than the reference scenario. Generally, compared to the reference (DM), the ICER for both DBT scenarios became more favourable with increasing DBT sensitivity.

\section{Scenario 1: DBT screening only for women with dense breasts}

DBT screening only for women with dense breasts was the most effective scenario when the sensitivity of DBT was at least $72 \%$ (Table 2). Compared with the reference, when a $3 \%$ discounting rate was applied, at a cost of $€ 96$, scenario 1 was cost-effective at a sensitivity of approximately $86 \%$ (Table 3 and Fig. 1a), whereas at a cost of $€ 80$, scenario 1 became costeffective when DBT sensitivity was at least $80 \%$. (Table 3 and Fig. 1b).

\section{Scenario 2: DBT for whole population}

At a DBT sensitivity of $80 \%$, the effectiveness of screening with DBT was comparable to screening with DM regarding the number of screen-detected cancers, interval cancers and LYGs (Table 2). When the sensitivity of DBT was $>85 \%$, scenario 2 was the most effective resulting in at least 26 more screen-detected cancers, 19 fewer interval cancers and 64 more undiscounted LYG per 10,000 women than biennial DM screening (Table 2). However, at a cost of $€ 96$, scenario 2 would not be cost-effective unless the sensitivity of DBT was larger than $95 \%$ when a $3 \%$ discounting rate was applied (Fig. 1a). However, if the cost of DBT decreased to $€ 80$, scenario 2 could be cost-effective when DBT sensitivity was around $90 \%$ (Fig. 1b). 
Table 2 Screening outcomes of using biennial DBT in a population screening program

\begin{tabular}{|c|c|c|c|c|c|c|c|c|}
\hline Sensitivity of DBT & $65 \%$ & $70 \%$ & $75 \%$ & $80 \%$ & $85 \%$ & $90 \%$ & $95 \%$ & $100 \%$ \\
\hline \multicolumn{9}{|c|}{ DBT for dense breasts compared to DM (scenario 1-reference) } \\
\hline$N$ screen-detected & $-12(0)$ & $-3(0)$ & $+6(0)$ & $+15(0)$ & $+24(1)$ & $+31(1)$ & $+40(1)$ & $+47(1)$ \\
\hline$N$ interval & $+12(0)$ & $+4(0)$ & $-4(0)$ & $-12(0)$ & $-19(0)$ & $-25(0)$ & $-32(1)$ & $-39(1)$ \\
\hline LYG & $-39(2)$ & $-14(2)$ & $+12(2)$ & $+40(4)$ & $+63(4)$ & $+88(5)$ & $+115(5)$ & $+138(5)$ \\
\hline Discounted $^{\mathrm{a}} \mathrm{LYG}$ & $-14(1)$ & $-5(1)$ & $+5(1)$ & $+19(2)$ & $+30(2)$ & $+42(2)$ & $+54(2)$ & $+65(2)$ \\
\hline Discounted ${ }^{\mathrm{b}} \mathrm{LYG}$ & $-23(1)$ & $-8(1)$ & $+8(2)$ & $+27(3)$ & $+43(3)$ & $+60(3)$ & $+78(3)$ & $+94(3)$ \\
\hline \multicolumn{9}{|c|}{ DBT for all women compared to DM (scenario 2-reference) } \\
\hline$N$ screen-detected & $-74(1)$ & $-47(1)$ & $-22(1)$ & $+2(1)$ & $+26(1)$ & $+48(0)$ & $+71(0)$ & $+91(1)$ \\
\hline$N$ interval & $+66(1)$ & $+43(1)$ & $+21(0)$ & $+1(1)$ & $-19(0)$ & $-38(1)$ & $-57(0)$ & $-74(1)$ \\
\hline LYG & $-219(3)$ & $-146(4)$ & $-76(3)$ & $-5(4)$ & $+64(4)$ & $+129(3)$ & $+195(4)$ & $+254(5)$ \\
\hline Discounted $^{\mathrm{a}} \mathrm{LYG}$ & $-76(1)$ & $-51(1)$ & $-25(1)$ & $+3(2)$ & $+31(2)$ & $+62(3)$ & $+92(2)$ & $+120(3)$ \\
\hline Discounted ${ }^{\mathrm{b}} \mathrm{LYG}$ & $-126(2)$ & $-84(2)$ & $-43(2)$ & $+1(3)$ & $+44(3)$ & $+88(4)$ & $+133(3)$ & $+172(4)$ \\
\hline \multicolumn{9}{|c|}{ DBT for all women compared to DBT for dense breasts (scenario 2 -scenario 1 ) } \\
\hline$N$ screen-detected & $-62(1)$ & $-44(1)$ & $-28(1)$ & $-13(0)$ & $+3(0)$ & $+17(1)$ & $+31(1)$ & $+44(1)$ \\
\hline$N$ interval & $+54(1)$ & $+39(0)$ & $+25(1)$ & $+12(0)$ & $0(0)$ & $-12(0)$ & $-24(1)$ & $-35(1)$ \\
\hline LYG & $-180(3)$ & $-132(4)$ & $-88(3)$ & $-45(2)$ & $+1(1)$ & $+41(2)$ & $+80(3)$ & $+117(3)$ \\
\hline Discounted $^{\mathrm{a}} \mathrm{LYG}$ & $-62(1)$ & $-46(1)$ & $-30(1)$ & $-16(1)$ & $+1(0)$ & $+19(1)$ & $+38(1)$ & $+54(1)$ \\
\hline Discounted $^{\mathrm{b}} \mathrm{LYG}$ & $-104(2)$ & $-76(2)$ & $-51(2)$ & $-26(1)$ & $+1(1)$ & $+28(1)$ & $+54(2)$ & $+79(2)$ \\
\hline
\end{tabular}

Data shown as differences in number of DBT screen-detected tumours ( $N$ screen-detected) and number of interval tumours $(N$ interval) for scenarios 1 and 2 with respect to the reference scenario of biennial DM screening for women 50-75 years of age, and for scenario 2 with respect to scenario 1. All data expressed as mean (SEs) per 10,000 women screened. Discounting ${ }^{\mathrm{a}}: 3 \%$ for both cost and LYG. Discounting ${ }^{\mathrm{b}}: 4 \%$ for cost and $1.5 \%$ for LYG $N=$ number; $L Y G=$ life years gained; $D M=$ digital mammography; $D B T=$ digital breast tomosynthesis.

Values in italics indicate the scenario ourperforms the comparison in the screening outcome

\section{Scenario 2 compared with scenario 1}

When DBT sensitivity was lower than $85 \%$, scenario 2 was always dominated by scenario 1 . At a cost of $€ 96$, scenario 2 could never be cost-effective even the sensitivity of DBT was $100 \%$ when discounted by international rate (Fig. 1a), whereas scenario 2 could be cost-effective than scenario $1 \mathrm{w}$ hen the sensitivity of DBT was around $95 \%$ at a cost of $€ 80$ (Fig. 1b).

\section{Discussion}

Our simulation modelling showed that for women aged 50 75 years, biennial screening with DBT for women with dense breasts is cost-effective when the sensitivity is around $90 \%$ (depending on the international discount rate), whereas DBT is unlikely to be a cost-effective alternative to DM if used to screen the general population when the cost of DBT is $€ 96$.
Table 3 The cost-effectiveness of biennial screening using DBT in a population screening program

\begin{tabular}{|c|c|c|c|c|c|c|}
\hline \multirow{2}{*}{$\begin{array}{l}\text { Sensitivity } \\
\text { of DBT }(\%)\end{array}$} & \multicolumn{3}{|c|}{ At $€ 96$ per DBT } & \multicolumn{3}{|c|}{ At $€ 80$ per DBT } \\
\hline & $\begin{array}{l}\text { Scenario } 1 \\
\text { compared to } \\
\text { reference }\end{array}$ & $\begin{array}{l}\text { Scenario } 2 \\
\text { compared to } \\
\text { reference }\end{array}$ & $\begin{array}{l}\text { Scenario } 2 \\
\text { compared to } \\
\text { scenario } 1\end{array}$ & $\begin{array}{l}\text { Scenario } 1 \\
\text { compared to } \\
\text { reference }\end{array}$ & $\begin{array}{l}\text { Scenario } 2 \\
\text { compared to } \\
\text { reference }\end{array}$ & $\begin{array}{l}\text { Scenario2 } \\
\text { compared to } \\
\text { scenario } 1\end{array}$ \\
\hline 65 & - & - & - & - & - & - \\
\hline 70 & - & - & - & - & - & - \\
\hline 75 & $180.3(37.4)$ & - & - & $91.1(18.9)$ & - & - \\
\hline 80 & $41.0(3.8)$ & - & - & $20.8(1.9)$ & - & - \\
\hline 85 & $24.4(1.6)$ & $63.7(4.5)$ & - & $12.4(0.8)$ & $32.2(2.3)$ & - \\
\hline 90 & $17.3(0.8)$ & $31.3(1.3)$ & $61.9(2.6)$ & $8.8(0.4)$ & $15.9(0.7)$ & $31.3(1.3)$ \\
\hline 95 & $13.2(0.5)$ & $20.8(0.5)$ & $31.9(0.9)$ & $6.7(0.2)$ & $10.6(0.3)$ & $16.2(0.5)$ \\
\hline 100 & $11.0(0.3)$ & $16.0(0.4)$ & $22.1(0.6)$ & $5.6(0.2)$ & $8.2(0.2)$ & $11.2(0.3)$ \\
\hline
\end{tabular}

Data are reported as incremental cost-effectiveness ratios (ICERs) with associated standard errors. ICERs are expressed as 1000 euros per LYG. A discount rate of $3 \%$ was applied to costs and life years gained. Data which not shown in the table indicates that the specific scenario was dominated by the comparator 
Fig. 1 The discounted incremental cost-effectiveness ratio (ICER) as a function of the sensitivity of digital breast tomosynthesis (DBT) at a DBT cost of $€ 96(\mathbf{a})$, or at a DBT cost of $€ 80$ (b). Discount rate of $3 \%$ was applied to costs and life years gained. Scenario 1: DBT for women with dense breast; scenario 2: DBT for whole population; reference: DM for whole population. Abbreviations: ICER $=$ incremental costeffectiveness ratio

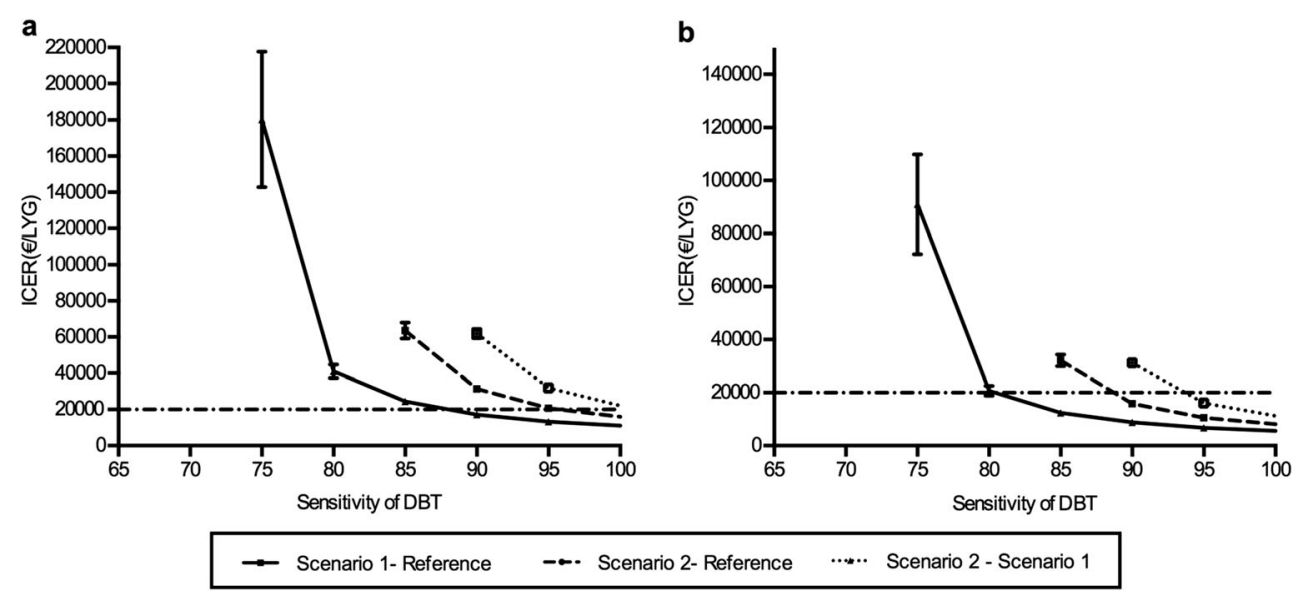

However, if the price of DBT decreased to $€ 80$, the sensitivities to be cost-effective would decrease to $80 \%$ and $90 \%$ for scenarios 1 and 2, respectively. Our findings may be of immediate relevance to policy makers considering population breast cancer screening programs with DBT, or those planning density-tailored screening scenarios.

Instead of using a point estimate for DBT sensitivity, we used a sensitivity range to estimate the lowest sensitivity at which DBT would be cost-effective in a population breast cancer screening program, given the range of published DBT sensitivities. In population-based screening programs, the performance of DBT showed a similar or slightly higher sensitivity compared to DM, and the DBT sensitivity was varied between 81.1 and $91.6 \%$ [12, 34-36]. A recent metaanalysis restricted to women with dense breasts showed that the sensitivity of DBT or DBT plus DM was higher (84-90\%) than DM alone (69-86\%) [37]. Given the range of sensitivity values, the required threshold sensitivity in our findings suggests that only when the cost of DBT was around $€ 80$, DBT could be a cost-effective alternative to DM when applied for screening the whole population at a sensitivity of $90 \%$. However, no matter which value of these two DBT costs is used, DBT screening only for women with dense breasts could be cost-effective, since the threshold for dense breasts is within the reported DBT sensitivity range. Another important issue is that previous studies have shown that DBT detects more tumours than DM $[36,38]$. Therefore, the sensitivity of DM is likely to be overestimated when cancers that are only detected by DBT are not taken into account (unless there has been adequate follow-up). However, in a recent study using DM and DBT in over 24,000 women where DBT-only cancers were also counted, the modality sensitivity of DM was approximately $80 \%$ which is comparable to the overall modality sensitivity of $79 \%$ used in our study [36]. Nevertheless, as it is likely that there might still be some overestimation of DM sensitivity, this might lead to a slight underestimation of DBT cost-effectiveness. We expect, however, that this would not influence the main outcomes of our study.
DBT for population screening is becoming more widespread in some settings, based on cancer detection and recall metrics; however, there is limited evidence on whether DBT could be a cost-effective alternative to DM in a population screening program, and little knowledge exists on its longterm outcomes. Only two studies have so far compared the cost-effectiveness of DBT plus DM to DM for breast cancer screening, both done in the USA $[10,39]$. Lee et al simulated biennial DBT plus DM among women aged 50-74 years with dense breasts using a discrete-event model, and the estimated sensitivity of DBT plus DM was $80 \%$, which was lower than our estimated threshold [10]. The ICER was $\$ 53,893$ which was considered to be cost-effective using a US threshold of $\$ 100,000$ per QALY [40]. In our study, using a DBT sensitivity of $80 \%$, at the cost of $€ 96$, the ICER for women with dense breasts was $€ 41,021$ per LYG, generally similar results to Lee et al [10]. Kalra et al simulated annual DBT plus DM among women aged 40 years and older using a Markov cohort decision-analytic model [39]. The ICER was \$20,230 per QALY, which was much lower than the ICER of screening the whole population as shown in our study.

The cost-effectiveness of DBT screening is highly dependent on the cost of DBT per examination. There are many factors that can influence DBT costs. Reading time is the main indirect cost related to DBT screening. Reading DBT images roughly doubles the interpretation time for mammograms [29]. Secondly, the price of new digital mammographic units with DBT capability entails an upfront investment [41]. A dedicated workstation is also needed to interpret DBT images, also requiring additional funding [41]. Information technology and image archiving infrastructure is substantially higher for DBT, for example DBT storage space can be 100 to 200 times that of DM [41]. In previous studies from the USA, the costs of adjunct DBT are found to be around 30-40\% higher compared to DM alone [10, 39], while the To-Be trial in Norway reported an incremental screening cost of $€ 8.5$ per screen [42]. However, the cost estimated in the To-Be trial might be underestimated. Firstly, the screening machines ran 
at a $100 \%$ capacity throughout the study period, which might not always be the case in practice. Secondly, the cost per screen depends on the number of women screened, and the trial was conducted in a city with a large population density, the cost might be higher when generalised to a population program. Therefore, in our study, we used two estimated values, a conventional estimate for the price of DBT at 50\% higher than DM, and a 25\% higher estimate based on expert opinion.

Regarding specificity, there is no consensus on whether DBT specificity is improved compared to DM. Some studies showed that specificity can be improved with adjunct DBT compared to DM alone [43, 44]. However, a meta-analysis addressed that the recall rate of adjunct DBT increased slightly in European countries, where the recall rate of DM was relatively low compared to other regions such as the USA [9]. In addition, the Malmö screening trial, a prospective study conducted in Sweden, reported an increase in false positive recall rate for one-view DBT compared with two-view DM (1.7\% vs $0.9 \%$, respectively) [12]. In a recent meta-analysis restricted to women with dense breasts, the improvement in specificity from DBT was found to be inconsistent in a screening setting [37]. Because of these inconsistent results, the specificity of DBT in our analysis was fixed at a conventional estimate value of $96.5 \%$, which was the same as the specificity of DM.

Our study used a validated model for which a sensitivity analysis has been done in a previous study indicating that the outcomes of the model were most sensitive to changes in the lifetime risk of breast cancer [17]. In our model, the sensitivity of DM depends on breast density and age, so increasing age generally leads to a decrease in breast density and to a corresponding increase in sensitivity of DM. This is an important component to consider as approximately $36 \%$ of Dutch women have dense breasts [45]. In addition, the model includes an increased risk of cancer induction due to radiation dose, which was not considered in previous studies that investigated the cost-effectiveness of DBT as a screening modality [10,39]. Although we used a relatively higher dose for DBT compared to $\mathrm{DM}$ ( $4 \mathrm{mGy}$ vs $3 \mathrm{mGy}$, respectively), only $0.4 \%$ more tumours were introduced by the increased dose. Therefore, we estimate that the influence of the increased dose from DBT has a negligible influence on the outcomes of our model.

As to limitations of the study, we point out that ductal carcinoma in situ is not included in this model. DCIS accounts for nearly $20 \%$ of the DM screen-detected tumours in the Dutch population and similar proportions are detected in other population screening programs $[18$, 46]. However, previous studies have shown that DBT plus DM can increase invasive CDR without preferentially increasing the proportion of DCIS compared to DM alone [47]. So, we expect that addition of DCIS into the model would not substantially change the major outcomes of the analysis. Another potential limitation is that we used values for DM sensitivity generated using meta-analysis [15]; this included some studies that were based on single-reading screening setting, suggesting that DM sensitivities might be lower compared to double-reading screening setting. The cost-effectiveness of DBT would be overestimated especially for scenario 2 , where DBT was used for the whole population. Thirdly, it has been shown that specificity increases with a decrease in breast density [22]. There is, however, no reliable data on the dependence of specificity on breast density for DBT. Therefore, as a first-order estimation, we used a constant specificity which was independent of breast density in our model. Nevertheless, if a similar decrease in specificity for both DBT and DM was applied in dense breasts, the ICER in our study would not be influenced as the number of false positives would be similar for both DBT and DM, making the incremental cost nearly unchanged. In addition, when implementing scenario 1 in practice, it is likely that every woman would get a DBT examination in the first screening round in order to assess breast density from the synthetic mammogram. This results in a $1.1 \%$ increase in total costs. Therefore, in order for scenario 1 to be costeffective, the sensitivity of DBT increases to $92 \%$ at a cost of $€ 96$ and approximately $85 \%$ at a cost of $€ 80$ per screen. Finally, we modelled the modality sensitivity of DBT, where we evaluated the effectiveness of a screening program. Because DBT is a relatively new technique, there are no data available on the sensitivity of a screening program including DBT, as long follow-up is needed to obtain reliable estimates of program sensitivity [28].

\section{Conclusion}

Several European countries, and Australia, have recently evaluated DBT in trials performed in population-based screening programs $[12,35,36,48]$. However, DBT is a relatively new technology, and whether DBT could be an alternative cost-effective replacement for DM in a population screening program is a matter of ongoing investigations. Based on our analysis, DBT is more likely to be a cost-effective alternative to DM in women with dense breasts. The threshold sensitivity for DBT to be costeffective is $90 \%$ and $80 \%$ at $€ 96$ and $€ 80$, respectively. Whether DBT could be cost-effective in a general population highly depends on the DBT cost. We envisage that a differential screening scenario in a population screening program (such as the proposed DBT only for dense breasts) requires innovative studies and trials to determine how this could be evaluated in pragmatic implementation studies. Such an approach would also require consideration of ethical issues and stakeholder consultation. 
Funding information The authors state that this work has not received any funding. J Wang receives support from Chinese scholarship council (CSC).

\section{Compliance with ethical standards}

Guarantor The scientific guarantor of this publication is Geertruida H.de Bock.

Conflict of interest The authors of this manuscript declare no relationships with any companies whose products or services may be related to the subject matter of the article. N Houssami receives research support through a National Breast Cancer Foundation (Australia) Breast Cancer Research Leadership Fellowship.

Statistics and biometry No complex statistical methods were necessary for this paper.

Informed consent Written informed consent was not required for this study because all eligible data was extracted from previous publications.

Ethical approval Institutional Review Board approval was not required because all eligible data was extracted from previous publications.

Methodology - Performed at one institution

Open Access This article is licensed under a Creative Commons Attribution 4.0 International License, which permits use, sharing, adaptation, distribution and reproduction in any medium or format, as long as you give appropriate credit to the original author(s) and the source, provide a link to the Creative Commons licence, and indicate if changes were made. The images or other third party material in this article are included in the article's Creative Commons licence, unless indicated otherwise in a credit line to the material. If material is not included in the article's Creative Commons licence and your intended use is not permitted by statutory regulation or exceeds the permitted use, you will need to obtain permission directly from the copyright holder. To view a copy of this licence, visit http://creativecommons.org/licenses/by/4.0/.

\section{References}

1. Curado MP, Edwards B, Shin HR et al (2007) Cancer incidence in five continents, Vol. IX, IARC Scientific Publications, No. 160, Lyon, IARC

2. Saadatmand S, Bretveld R, Siesling S, Tilanus-Linthorst MM (2015) Influence of tumour stage at breast cancer detection on survival in modern times: population based study in 173,797 patients. BMJ 351:h4901

3. De LM, Fracheboud J, De GB, Den GH, Siesling S, Broeders M (2018) Is the incidence of advanced-stage breast cancer affected by whether women attend a steady-state screening program? Int J Cancer 143:842-850

4. Houssami N, Miglioretti DL (2016) Breast cancer screening: an examination of scientific evidence. Elsevier, London

5. Chen HH, Yen MF, Fann CY et al (2017) Clarifying the debate on population-based screening for breast cancer with mammography: a systematic review of randomized controlled trials on mammography with Bayesian meta-analysis and causal model. Medicine (Baltimore) 96:e5684
6. Bae MS, Moon WK, Chang JM (2014) Breast cancer detected with screening US: reasons for nondetection at mammography. Radiology 270:369-377

7. Nelson HD, O'Meara ES, Kerlikowske K, Balch S, Miglioretti D (2016) Factors associated with rates of false-positive and falsenegative results from digital mammography screening: an analysis of registry data. Ann Intern Med 164:226-235

8. Tagliafico AS, Mariscotti G, Valdora F et al (2018) A prospective comparative trial of adjunct screening with tomosynthesis or ultrasound in women with mammography-negative dense breasts (ASTOUND-2). Eur J Cancer 104:39-46

9. Marinovich ML, Hunter KE, Macaskill P, Houssami N (2018) Breast cancer screening using tomosynthesis or mammography: a meta-analysis of cancer detection and recall. J Natl Cancer Inst 110: 942-949

10. Lee CI, Cevik M, Alagoz O (2015) Comparative effectiveness of combined digital mammography and tomosynthesis screening for women with dense breasts. Radiology 274:772-780

11. Zuckerman SP, Maidment ADA, Weinstein SP, McDonald ES, Conant EF (2017) Imaging with synthesized 2D mammography: differences, advantages, and pitfalls compared with digital mammography. AJR Am J Roentgenol 209:222-229

12. Zackrisson S, Lang K, Rosso A et al (2018) One-view breast tomosynthesis versus two-view mammography in the Malmo Breast Tomosynthesis Screening Trial (MBTST): a prospective, population-based, diagnostic accuracy study. Lancet Oncol 19: 1493-1503

13. Bernardi D, Belli P, Benelli E et al (2017) Digital breast tomosynthesis (DBT): recommendations from the Italian College of Breast Radiologists (ICBR) by the Italian Society of Medical Radiology (SIRM) and the Italian Group for Mammography Screening (GISMa). Radiol Med 122:723-730

14. Husereau D, Drummond M, Petrou S et al (2013) Consolidated health economic evaluation reporting standards (CHEERS) statement. Value Health 16:e1-e5

15. Koleva-Kolarova RG, Daszczuk AM, de Jonge C et al (2018) A modelling study to evaluate the costs and effects of lowering the starting age of population breast cancer screening. Maturitas 109: $81-88$

16. de Bock GH, Vermeulen KM, Jansen L et al (2013) Which screening strategy should be offered to women with BRCA1 or BRCA2 mutations? A simulation of comparative cost-effectiveness. Br J Cancer 108:1579-1586

17. Greuter MJ, Jansen-van der Weide MC, Jacobi CE et al (2010) The validation of a simulation model incorporating radiation risk for mammography breast cancer screening in women with a hereditary-increased breast cancer risk. Eur J Cancer 46:495-504

18. LETB (2014) National evaluation of breast cancer screening in the Netherlands 1990-2011/2012

19. Cijfers over kanker(2017) Available from: http://www. cijfersoverkanker.nl/. Accessed on 23th April 2018

20. Checka CM, Chun JE, Schnabel FR, Lee J, Toth H (2012) The relationship of mammographic density and age: implications for breast cancer screening. AJR Am J Roentgenol 198:W292-W295

21. Kerlikowske K, Grady D, Barclay J, Sickles EA, Ernster V (1996) Effect of age, breast density, and family history on the sensitivity of first screening mammography. JAMA 276:33-38

22. Lehman CD, White E, Peacock S, Drucker MJ, Urban N (1999) Effect of age and breast density on screening mammograms with false-positive findings. AJR Am J Roentgenol 173:1651-1655

23. Peer PG, van Dijck JA, Hendriks JH, Holland R, Verbeek AL (1993) Age-dependent growth rate of primary breast cancer. Cancer 71:3547-3551

24. Skaane P, Hofvind S, Skjennald A (2007) Randomized trial of screen-film versus full-field digital mammography with soft-copy 
reading in population-based screening program: follow-up and final results of Oslo II study. Radiology 244:708-717

25. Destounis S, Johnston L, Highnam R, Arieno A, Morgan R, Chan A (2017) Using volumetric breast density to quantify the potential masking risk of mammographic density. AJR Am J Roentgenol 208:222-227

26. Skaane P, Bandos AI, Gullien R et al (2013) Prospective trial comparing full-field digital mammography (FFDM) versus combined FFDM and tomosynthesis in a population-based screening programme using independent double reading with arbitration. Eur Radiol 23:2061-2071

27. Sankatsing VD, Heijnsdijk EA, van Luijt PA, van Ravesteyn NT, Fracheboud J, de Koning HJ (2015) Cost-effectiveness of digital mammography screening before the age of 50 in the Netherlands. Int J Cancer 137:1990-1999

28. Hakama M, Auvinen A, Day NE, Miller AB (2007) Sensitivity in cancer screening. J Med Screen 14:174-177

29. Skaane P, Bandos AI, Gullien R et al (2013) Comparison of digital mammography alone and digital mammography plus tomosynthesis in a population-based screening program. Radiology 267:47-56

30. Zorginstituut Nederland (2015) Rapport Kosteneffectiviteit in de praktijk. Available from https://www.zorginstituutnederland.nl. Accessed on 6 Nov 2016

31. World Health Organization (2003) Making choices in health: WHO guide to cost-effectiveness analysis. World Health Organization Geneva. Available via https://www.who.int/choice/publications/p_ 2003 generalised_cea.pdf. Accessed on 26 Aug 2019

32. Lipscomb J, Weinstein MC, Torrance GW (1996) Time preference. In: Gold MR, Seigel JE, Russell LB, Weinstein MC (eds) Costeffectiveness in health and medicine. Oxford University Press, New York

33. Healthcare Institute of the Netherlands (ZIN) (2015) Richtlijn voor economische evaluaties van zorginterventies. Available from www. zorginstituutnederland.nl. Accessed on 11 Jan 2016

34. Conant EF, Barlow WE, Herschorn SD et al (2019) Association of digital breast tomosynthesis vs digital mammography with cancer detection and recall rates by age and breast density. JAMA Oncol 5: 635-664

35. Houssami N, Bernardi D, Caumo F et al (2018) Interval breast cancers in the 'screening with tomosynthesis or standard mammography' (STORM) population-based trial. Breast 38:150-153

36. Skaane P, Bandos AI, Niklason LT et al (2019) Digital mammography versus digital mammography plus tomosynthesis in breast cancer screening: the Oslo Tomosynthesis Screening Trial. Radiology 291:23-30
37. Phi XA, Tagliafico A, Houssami N, Greuter MJW, de Bock GH (2018) Digital breast tomosynthesis for breast cancer screening and diagnosis in women with dense breasts - a systematic review and meta-analysis. BMC Cancer 18:380

38. Skaane P, Sebuodegard S, Bandos AI et al (2018) Performance of breast cancer screening using digital breast tomosynthesis: results from the prospective population-based Oslo Tomosynthesis Screening Trial. Breast Cancer Res Treat 169:489-496

39. Kalra VB, Wu X, Haas BM, Forman HP, Philpotts LE (2016) Costeffectiveness of tomosynthesis in annual screening mammography. AJR Am J Roentgenol 207:1152-1155

40. Neumann PJ, Cohen JT, Weinstein MC (2014) Updating costeffectiveness-the curious resilience of the $\$ 50,000$-per-QALY threshold. N Engl J Med 371:796-797

41. Patterson SK, Roubidoux MA (2014) Update on new technologies in digital mammography. Int J Womens Health 6:781-78843

42. Moger TA, Swanson JO, Holen ÅS, Hanestad B, Hofvind S (2019) Cost differences between digital tomosynthesis and standard digital mammography in a breast cancer screening programme: results from the To-Be trial in Norway. Eur J Health Econ 20:1261-1269

43. Haas BM, Kalra V, Geisel J, Raghu M, Durand M, Philpotts LE (2013) Comparison of tomosynthesis plus digital mammography and digital mammography alone for breast cancer screening. Radiology 269:694-700

44. Hofvind S, Holen ÅS, Aase HS et al (2019) Two-view digital breast tomosynthesis versus digital mammography in a population-based breast cancer screening programme (To-Be): a randomised, controlled trial. Lancet Oncol 20:795-805

45. Wanders JO, Holland K, Veldhuis WB et al (2017) Volumetric breast density affects performance of digital screening mammography. Breast Cancer Res Treat 162:95-103

46. Weigel S, Khil L, Hense HW et al (2018) Detection rates of ductal carcinoma in situ with biennial digital mammography screening: radiologic findings support pathologic model of tumor progression. Radiology 286:424-432

47. Caumo F, Romanucci G, Hunter K et al (2018) Comparison of breast cancers detected in the Verona screening program following transition to digital breast tomosynthesis screening with cancers detected at digital mammography screening. Breast Cancer Res Treat 170:391-397

48. Houssami N, Lockie D, Clemson M et al (2019) Pilot trial of digital breast tomosynthesis (3D mammography) for population-based screening in BreastScreen Victoria. Med J Aust 211:357-362

Publisher's note Springer Nature remains neutral with regard to jurisdictional claims in published maps and institutional affiliations. 